\title{
Nuclear Pasta Structure in Hot Neutron Stars
}

\author{
Gentaro Watanabe $e^{1,2,3}$, Katsuhiko Sato ${ }^{1,4}$, Kenji Yasuoka ${ }^{5}$ and \\ Toshikazu Ebisuzaki ${ }^{2}$ \\ ${ }^{1}$ Department of Physics, University of Tokyo, Tokyo 113-0033, Japan \\ ${ }^{2}$ RIKEN, Saitama 351-0198, Japan \\ ${ }^{3}$ NORDITA, Blegdamsvej 17, DK-2100 Copenhagen $\emptyset$, Denmark \\ ${ }^{4}$ RESCEU, University of Tokyo, Tokyo 113-0033, Japan \\ ${ }^{5}$ Department of Mechanical Engineering, Keio University, Yokohama \\ 223-8522, Japan
}

\begin{abstract}
The structure of cold and hot dense matter at subnuclear densities is investigated by quantum molecular dynamics simulations. The phase diagrams obtained show that the density of the phase boundaries between the different nuclear structures decreases with increasing temperature due to the thermal expansion of the nuclear matter region.
\end{abstract}

\section{Introduction}

At subnuclear densities, nuclear matter exhibits the coexistence of a liquid phase with a gas phase. Just below the density where nuclei melt into uniform matter, it is expected that, at sufficiently low temperatures $(T \ll 1 \mathrm{MeV})$, the energetically favorable configuration of the mixed phase possesses interesting spatial structures such as rodlike and slablike nuclei, etc, which are called nuclear "pasta" (Hashimoto et al. 1984; Ravenhall et al. 1983). While nuclear "pasta" at zero temperature has been studied by several authors, "pasta" phases at finite temperatures relevant to supernova cores and crusts of young hot neutron stars have not been studied yet except for some limited cases (Lassaut et al. 1987; Watanabe et al. 2001, 2003a).

\section{New Results}

Here we study the structure of hot dense matter at subnuclear densities by quantum molecular dynamics. Simulations of the $(n, p, e)$ system at proton fractions of $x=0.3$ and 0.5 are performed with 2048 nucleons in a cubic periodic box. The relativistic degenerate electrons are regarded as a uniform background and the Coulomb interaction is calculated by the Ewald method. The effective Hamiltonian used in this work is that developed by Maruyama et al. (1998).

We show the resultant phase diagram for $x=0.5$ on the nucleon density $\rho$ vs temperature $T$ plane in Figure 1. The phase separation line is determined by the disappearance of the long-range correlation of the nucleon distribution detected by the two-point correlation function. The nuclear surface is identified by the plateau of the Euler characteristic density $\chi / V$ as a function of threshold density for the isodensity surface. In the density region of interest, the nuclear surface 
cannot be observed typically at $T \gtrsim 3 \mathrm{MeV}$, even in the phase-separating region. At temperatures where the nuclear surface can be identified, we characterize the nuclear shape changes with increasing density by using the averaged mean curvature $\langle H\rangle$ and $\chi / V$ : (a) $\langle H\rangle>0, \chi / V>0 \rightarrow$ (b) $\langle H\rangle>0, \chi / V=0 \rightarrow$ (c) $\langle H\rangle>0, \chi / V<0 \rightarrow$ (d) $\langle H\rangle=0, \chi / V=0 \rightarrow$ (e) $\langle H\rangle<0, \chi / V<0 \rightarrow$ (f) $\langle H\rangle<0, \chi / V=0 \rightarrow$ (g) $\langle H\rangle<0, \chi / V>0 \rightarrow$ uniform. The critical point for the phase separation is located at $\rho \sim 0.25 \rho_{0}$ and $T \gtrsim 6 \mathrm{MeV}$. We note that the density of each phase boundary between different structures decreases as $T$ increases, which is due to thermal expansion of the nuclear matter region.

Our result suggests that "pasta" phases, and in addition, "spongelike" phases with negative values of $\chi / V$, exist' in hot neutron star crusts and supernova cores at $T \lesssim 3 \mathrm{MeV}$.

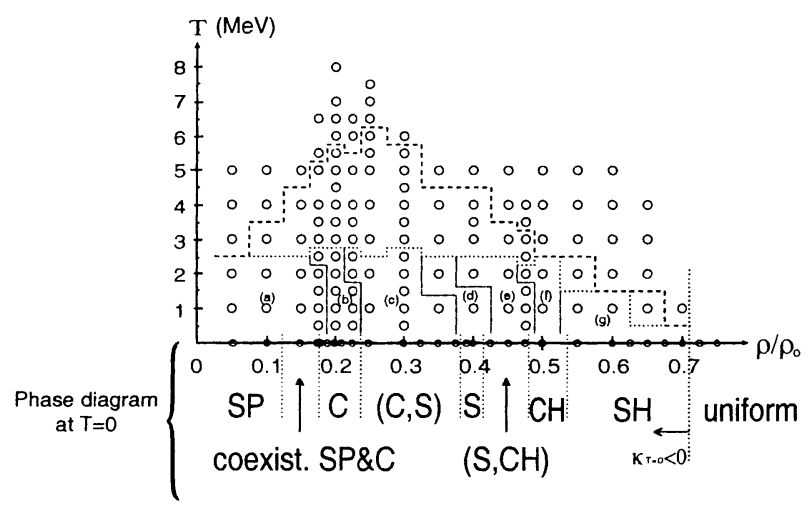

Figure 1. Phase diagram at $x=0.5$. The dashed and dotted lines on the diagram show the phase separation line and the limit below which the nuclear surface can be identified, respectively. The dash-dotted lines are the phase boundaries between the different nuclear shapes. The symbols SP, C, S, CH and SH stand for nuclear shapes, i.e. sphere, cylinder, slab, cylindrical hole and spherical hole, respectively. The parentheses (A, B) show "spongelike" intermediate phases between Aand B-phases suggested in our previous works at $T=0$ (Watanabe et al. 2002, 2003b); they have negative $\chi / V$. Simulations have been carried out at points denoted by circles. For regions (a)-(g), see text.

\section{References}

Hashimoto, M. et al. 1984, Prog. Theor. Phys., 71, 320

Lassaut, M. et al. 1987, A\&A, 183, L3

Maruyama, T. et al. 1998, Phys. Rev. C, 57, 655

Ravenhall, D. G. et al. 1983, Phys. Rev. Lett., 50, 2066

Watanabe, G. et al. 2001, Nucl. Phys. A, 687, 512

- 2002, Phys. Rev. C, 66, 012801(R)

- 2003a, Nucl. Phys. A, 726, 357

- 2003b, Phys. Rev. C, 68, 035806 